\title{
Use of short term video EEG in the diagnosis of attack disorders
}

\author{
A McGonigal, A J C Russell, A K Mallik, M Oto, R Duncan
}

J Neurol Neurosurg Psychiatry 2004;75:771-772. doi: 10.1136/jnnp.2003.024893

Background: Distinguishing epileptic from psychogenic nonepileptic seizures (PNES) often requires video electroencephalography (EEG) recording. Inpatient recording is a limited resource; some evidence suggests that short term video EEG (SVEEG) is useful, but its role in practice has yet to be evaluated.

Objective: To assess the usefulness of SVEEG in the diagnosis of attack disorders.

Methods: One hundred and forty three SVEEG recordings were performed during an 18 month period.

Results: A diagnostic event was recorded in 72 of 143 (50.3\%): PNES ( $n=51$ ), epilepsy ( $=7)$, or other attacks, such as movement disorders ( $\mathrm{n}=14$ ).

Conclusions: SVEEG is a robust and useful diagnostic technique, which complements existing resources.

$\mathrm{T}$ he diagnosis of attack disorders can be difficult, and misdiagnosis of epilepsy is common. ${ }^{1}$ Distinguishing epileptic seizures from psychogenic non-epileptic seizures (pseudoseizures; PNES) relies on a detailed history from the patient and eyewitness, but recording typical attacks with inpatient video electroencephalography (video EEG) is frequently required. Access to this expensive resource is limited, with waiting lists of many months in some centres.

Short duration outpatient video EEG has been used successfully in paediatric practice. ${ }^{2}$ A recent study of adult patients has shown that habitual PNES can be reliably recorded during short outpatient video EEG in 50\% of patients, avoiding the need for inpatient video EEG in some. ${ }^{3}$ The wider clinical usefulness of this method has yet to be determined.

Early diagnosis of PNES may improve outcome, ${ }^{4}$ and avoids inappropriate anti-epileptic drug prescription. A means of diagnosing some patients earlier with a readily available and less costly investigation has important clinical and economic implications.

\section{AIMS}

To document the use and diagnostic yield of short term video EEG (SVEEG) as used in our unit over an 18 month period.

\section{BACKGROUND}

Our regional epilepsy service has two inpatient video EEG beds, serving a population of approximately 2.4 million in the west of Scotland, with additional referrals from other regions. Eighty to 90 patients are admitted to the unit each year. The mean waiting time for inpatient video EEG is nine to 12 months.

We previously carried out a pilot study of outpatient SVEEG in suspected PNES, ${ }^{3}$ since which the technique has been in clinical use. When PNES are suspected, or when an attack of some other type might be recorded (for example, frequent attacks or specific triggers), simultaneous video recording is performed. Therefore, the patients described here have been selected because of a clinical assessment that attacks may occur. SVEEG recordings are performed within four to 12 weeks of referral, or sooner depending on the clinical indication.

\section{METHODS}

Clinical neurophysiology medical staff interviewed each patient, to establish the clinical description of the attack, particularly whether one or more types of attack occurred. All patients were asked to bring an eyewitness if possible; if present at the time of recording, their account of the attack was also obtained.

A standard 16 channel EEG with single channel electrocardiogram and simultaneous digital video was recorded, with an average recording duration of 40-50 minutes. If PNES were suspected, simple suggestion techniques were used. $^{3}$ If an attack was recorded, it was shown to the eyewitness, to confirm whether typical; if no eyewitness was available at that time, the video was reviewed later. A diagnosis was made only if the recorded attack and EEG findings were unequivocal, and consistent with the available history. If doubt remained, or if not all types of attacks had been recorded, further monitoring was recommended.

\section{RESULTS}

\section{SVEEG}

One hundred and forty three SVEEG recordings were performed for the investigation of attack disorders during an 18 month period (July 2000 to December 2001). During this period, an additional 15 patients underwent SVEEG as part of a separate randomised controlled trial that has already been reported ${ }^{3}$; these patients were therefore not included in our study. The age range was 14-75 years; 47 patients were male and 96 female.

An attack was recorded in 83 of 143 recordings (table 1). Eleven of these were not clearly confirmed as being typical of the patient's usual event, and were classed as inconclusive.

Table 1 Type of attack recorded in 143 short term video EEG recordings, 2000-2001

\begin{tabular}{lc}
\hline Type of attack recorded & Number \\
\hline PNES & 51 \\
Epilepsy & 7 \\
Other "not epilepsy" & 14 \\
Inconclusive attack & 11 \\
No attack recorded & 60 \\
Total & 143 \\
\hline EEG, electroencephalography; PNES, psychogenic non- \\
epileptic seizures.
\end{tabular}

Abbreviations: EEG, electroencephalography; PNES, psychogenic nonepileptic seizures; SVEEG, short term video electroencephalography 
Table 2 Diagnostic outcome after inconclusive short term video EEG $(n=60)$

\begin{tabular}{lll}
\hline & Outcome & Number of patients \\
\hline $\begin{array}{l}\text { Further monitoring } \\
(\mathrm{n}=20)\end{array}$ & $\begin{array}{l}\text { On further monitoring, diagnostic events } \\
\text { were recorded* } \\
\text { On further monitoring, no events were recorded } \\
\text { but a clinical diagnosis reached } \\
\text { On further monitoring, no events were recorded; } \\
\text { the clinical diagnosis remains unclear and the } \\
\text { patient is under review }\end{array}$ & 8 \\
$\begin{array}{l}\text { Further monitoring awaited } \\
\text { Clinical diagnosis reached, further monitoring } \\
\text { felt to be unnecessary } \\
\text { No further monitoring planned (events too infrequent } \\
\text { or patient unsuitable for monitoring); diagnosis } \\
\text { unclear, patient under review }\end{array}$ & 5 \\
$\begin{array}{l}\text { Patient declined/did not attend for clinic follow } \\
\text { up/further monitoring }\end{array}$ & 28 \\
\hline *Diagnosis after event recorded on further monitoring: 4 patients had epilepsy; 3 had PNES plus epilepsy; 1 had \\
$\begin{array}{l}\text { PNES alone. } \\
\text { EEG, electroencephalography; PNES, psychogenic non-epileptic seizures. }\end{array}$
\end{tabular}

Therefore, a diagnostic event was recorded in 72 of 143 $(50.3 \%)$.

Epileptic events were recorded in seven patients: complex partial seizures in two, myoclonic jerks in four, and one generalised tonic clonic seizure. The "not epilepsy" group included movement disorders and hyperventilation related symptoms.

Eleven patients who had no attack recorded had a clinical diagnosis suggested by history and EEG data: in 10 this was epilepsy, and in one probable cough syncope. Appropriate specialist follow up was arranged.

Therefore, the total number of SVEEG recordings providing diagnostic information, either from recorded typical events $(\mathrm{n}=72)$ or history/EEG data $(\mathrm{n}=11)$, was 83 of 143 $(58 \%)$. The remaining 60 recordings were classed as "inconclusive".

\section{Outcome after "inconclusive" SVEEG}

After inconclusive SVEEG, 38 of 60 patients have since had a diagnosis made (table 2). An appreciable number of patients declined or did not attend for clinic review and/or further monitoring (seven of 60).

Further monitoring (videotelemetry and/or ambulatory EEG) was carried out, or is awaited, in 20 of 60 in the "inconclusive" group (table 2); some have had monitoring more than once. The long wait for monitoring in two patients reflects previous failure to attend for the appointment. Further monitoring has also been performed in seven patients with a previous diagnostic SVEEG who have more than one attack type, or who subsequently reported a change in seizure type.

\section{DISCUSSION}

SVEEG recording provided a useful yield of recorded attacks in this large series of patients, with habitual diagnostic events positively identified in 50\%. Some recordings where no attacks were captured yielded diagnostic information based on history and EEG alone. In these patients, the same information could of course have been gained by clinical consultation and interictal EEG.

Follow up of the original study group ${ }^{3}$ at one year indicates that the diagnostic information obtained during SVEEG is robust, with no diagnosis of PNES having been revised (unpublished data, 2002).

It is not possible to calculate accurately the proportion of patients who would otherwise have required inpatient video EEG, or the number of "bed days" saved, because not all of the patients who had SVEEG would have required inpatient recording. However, the advantages of SVEEG in terms of earlier diagnosis and lower cost are likely to be considerable.

Although the technique itself is relatively simple and practicable, we would not recommend its use where appropriate clinical and electrophysiological expertise is not available. Expert knowledge of attack disorders and ictal video EEG recording is required. The EEG may be obscured by artefact during attacks, and some types of epileptic seizure may not show changes on surface EEG. It is crucial to obtain evidence that the patient's habitual type of attack has been recorded, and to perform further recording if there is more than one type of event.

The diagnosis of attack disorders is a difficult and common clinical problem. The correct and timely diagnosis of some types of attacks may improve outcome, ${ }^{4}$ and saves medical costs. ${ }^{5}$ SVEEG is a useful diagnostic tool, particularly for suspected PNES, allowing prompt diagnosis and avoiding the need for inpatient video EEG in some patients. The technique complements existing investigative facilities, and may allow better use of the limited and expensive resource of inpatient video EEG.

\section{Authors' affiliations}

A McGonigal, A J C Russell, A K Mallik, M Oto, R Duncan, Regional Epilepsy Service, Institute of Neurology, Southern General Hospital, Glasgow G51 4TF, UK

Competing interest: none declared

Correspondence to: Dr A McGonigal, Regional Epilepsy Service, Institute of Neurology, Southern General Hospital, Glasgow G51 4TF, UK; aileenmcg@hotmail.com

Received 1 August 2003

Accepted 16 September 2003

\section{REFERENCES}

1 Chadwick D, Smith D. The misdiagnosis of epilepsy [editorial]. BMJ 2002;324:495-6.

2 Del Giudice E, Crisanti AF, Romano A. Short duration outpatient video electroencephalographic monitoring: the experience of a southern-Italian general pediatric department. Epileptic Disord 2002;3:197-202.

3 McGonigal A, Oto M, Russell AJC, et al. Outpatient video-EEG recording in the diagnosis of non-epileptic seizures: a randomised trial of simple suggestion techniques. J Neurol Neurosurg Psychiatry 2002:72:549-51

4 Walczak TS, Papacostas S, Williams DT. Outcome after diagnosis of psychogenic nonepileptic seizures. Epilepsia 1995;36:1131-7.

5 Martin RC, Gilliam FG, Kilgore M, et al. Improved health care resource utilization following video-EEG-confirmed diagnosis of nonepileptic psychogenic seizures. Seizure 1998;7:385-90. 